\title{
Site-Specific Secretome Map Evidences VSMC-Related Markers of Coronary Atherosclerosis Grade and Extent in the Hypercholesterolemic Swine
}

\author{
Silvia Rocchiccioli, ${ }^{1}$ Antonella Cecchettini, ${ }^{1,2}$ Nadia Ucciferri, ${ }^{1}$ \\ Marianna Terreni, ${ }^{1}$ Federica Viglione, ${ }^{1}$ Maria Giovanna Trivella, ${ }^{1}$ \\ Lorenzo Citti, ${ }^{1}$ Oberdan Parodi, ${ }^{1}$ and Gualtiero Pelosi ${ }^{1}$ \\ ${ }^{1}$ National Research Council, Institute of Clinical Physiology, Via Moruzzi, 56124 Pisa, Italy \\ ${ }^{2}$ Department of Clinical and Experimental Medicine, University of Pisa, Via Volta, 56126 Pisa, Italy \\ Correspondence should be addressed to Oberdan Parodi; oberdan.parodi@virgilio.it
}

Received 8 February 2015; Revised 29 April 2015; Accepted 23 June 2015

Academic Editor: Andreas Pich

Copyright (C) 2015 Silvia Rocchiccioli et al. This is an open access article distributed under the Creative Commons Attribution License, which permits unrestricted use, distribution, and reproduction in any medium, provided the original work is properly cited.

\begin{abstract}
A major drawback in coronary atherosclerosis (ATS) research is the difficulty of investigating early phase of plaque growth and related features in the clinical context. In this study, secreted proteins from atherosclerotic coronary arteries in a hypercholesterolemic swine model were characterized by a proteomics approach and their expression was correlated to site-specific ATS stage and extent. A wide coronary artery map of secreted proteins has been obtained in high fat (HF) diet induced ATS swine model and a significantly different expression of many proteins related to vascular smooth muscle cell (VSMC) activation/migration has been identified. Significant associations with ATS stage of HF coronary lesions were found for several VSMC-derived proteins and validated for chitinase 3 like protein 1 (CHI3L1) by tissue immunoexpression. A direct correlation $\left(R^{2}=0.85\right)$ was evidenced with intima to media thickness ratio values and ELISA confirmed the higher blood concentrations of CHI3L1 in HF cases. These findings confirmed the pivotal role of VSMCs in coronary plaque development and demonstrated a strong site-specific relation between VSMC-secreted CHI3L1 and lesion grade, suggesting that this protein could be proposed as a useful biomarker for diagnosing and staging of atherosclerotic lesions in coronary artery disease.
\end{abstract}

\section{Introduction}

Coronary atherosclerosis (ATS) is the underlying pathology of major acute events associated with thrombotic obstruction of vessel lumen and a leading cause of morbidity and mortality in Western countries [1,2].

Identification of molecular factors associated with coronary atherogenesis (i.e., coronary plaque formation and early progression) has relevant implications for diagnosis, prevention, and treatment of overt coronary artery disease (CAD), especially when significant associations emerge between these factors and the ATS grade, lesion extent, and progression.

Within this context, proteome profiling represents an interesting approach to disclose a broad spectrum of cellular proteins and peptides and to propose putative biomarkers [3]. A major obstacle in investigating coronary ATS initiation and progression is related to the advanced stage of human atherosclerotic samples collected at surgery and the consequent difficulties in digging putative biomarkers in a complex tissue proteome, characterized by a wide dynamic range of concentration. The study of secreted factors in atherosclerosis research could override the limits of a plaque proteome [4] evidencing hidden actors and representing a valuable source of noninvasive markers detectable in blood.

In order to reduce sample complexity in proteome studies and to obtain human tissue representatives of the early stages of plaque formation and progression, de la Cuesta et al. have recently [5] analyzed the medial layer of atherosclerotic coronary arteries by 2D-DIGE, highlighting a differential 
expression of several cytoskeleton proteins of atheromaadjacent VSMCs as compared to preatherosclerotic cells, and hypothesized a key role in plaque formation by activated VSMCs.

The same authors [6] have previously used a label-free liquid chromatography approach coupled with mass spectrometry (LC-MS/MS) analyses to compare the secretome data from human atherosclerotic coronary arteries with preatherosclerotic coronaries and intact mammaries (collected at autopsy) and identified four VSMC-related proteins, which were downreleased in the pathological samples.

Both proteomics approaches highlighted the key role of arterial VSMCs in atherosclerotic plaque initiation and growth. Moreover, this role was related to the well-known VSMC phenotypic heterogeneity, which accounts for various cell activities such as proliferation, migration, and synthesis/degradation of extracellular matrix (ECM) components [7]. Indeed, according to the classical theory of atherogenesis, after inflammatory cells and mediators initiate the process by foam cell formation, the overexpression of ECM proteoglycans (ECM-PGs), synthetized by activated VSMCs, would enhance subendothelial lipoprotein trapping while VSMC proliferation contributed to plaque growth and to its evolution towards stability [8].

A clear association of the full spectrum of lesion stages in coronary atherogenesis with known or novel molecular factors of pathology progression is currently missing for several reasons: (i) the inherent difficulty to investigate the early stage of ATS [9] and to identify low-abundant cellsecreted proteins; (ii) the limited number of studies which have focused on protein expression in the intima layer of early coronary lesions in humans and the even lower number of those that have compared preatherosclerotic with advanced coronary lesions [10-13]; (iii) the difficulty of assessing which of the observed coronary preatherosclerotic lesions, such as diffuse intimal thickening and fatty streaks, are prone to progress into advanced lesions and which will remain stable over decades, a condition that limits the identification of informative markers of evolving lesions [9].

All these limitations in studying plaque onset and formation have encouraged the use of animal models, among which swine is considered the closest to human. [14]. Although genomics and transcriptomics atherogenesis-related changes are reported in mouse and swine models $[15,16]$, a wide proteomics profiling of porcine coronary arteries, to identify molecular factors and protein pathways involved in plaque initiation and early formation, has not yet been described.

Aims of the present study are (i) to identify a molecular map of proteins secreted by intact and atherosclerotic coronary arteries of pigs fed on standard (CTRL) and hypercholesterolemic (HF) diet, respectively, using a hypothesis-free approach; (ii) to compare protein expressions of CTRL and HF samples and to evidence differentially secreted proteins that could be related to CAD onset and progression; and (iii) to associate the most relevant differentially expressed proteins with histomorphometrically characterized atherosclerotic lesions and to identify their cellular localization by immunohistochemistry.

\section{Methods}

\subsection{Experimental Protocol}

2.1.1. Animals and Diet. The protocol was applied to 12 male domestic pigs of 8 to 12 weeks of age. Animals were allocated into two groups: controls fed on standard chow (CTRL, $n=6$ ) and animals fed on high fat cholesterol-enriched diet (HF, $n=6$ ) for 4 months (119 days). Mean baseline body weight in the two groups was not significantly different and raised to $42 \pm 7 \mathrm{Kg}$ and $49 \pm 7 \mathrm{Kg}$ in CTRL and HF, respectively, at the end of diet period (mean values \pm SD, NS). High fat diet, as compared to standard one, was supplemented with $20 \%$ lard and $4 \%$ cholesterol $(4450 \mathrm{Kcal} / \mathrm{kg}$ with $54.6 \%$ of total energy provided by fat). The plasma lipid profile was evaluated: plasma triglycerides (TGs), high-density lipoprotein (HDL), low-density lipoprotein (LDL), and total cholesterol (TC) content were measured by enzymatic colorimetric reactions using commercial kits (Synchron CX9 Pro, Beckman Coulter Inc., USA). Values (mg/dL) in the CTRL group $(N=6$, mean \pm SD) were as follows: TGs: $29.5 \pm 19.3$; TC: $58.3 \pm 4.9$; HDL: $25.5 \pm 4.4$; LDL: $27 \pm 5.5$; TC/HDL ratio: $2.3 \pm 0.2$; values in HF group $(N=6$, mean \pm SD) were as follows: TGs: $65 \pm 45$; TC: $558 \pm 134$; HDL: $34 \pm 14$; LDL: $511.2 \pm$ 133.6; TC/HDL ratio: $19 \pm 7$. LDL was calculated according to Friedewald et al. [17]. Apolipoprotein A1 was measured by rate nephelometry (BN-ProSpec, Siemens Healthcare Diagnostics, Italy): CTRL group $(N=6$, mean $\pm \mathrm{SD}): 22.8 \pm 8.5 \mathrm{mg} / \mathrm{dL}$; HF group $(N=6$, mean $\pm \mathrm{SD}): 54.7 \pm 6.4 \mathrm{mg} / \mathrm{dL}$. All values were significantly different between CTRL and HF group.

2.1.2. Surgery. Anaesthesia was induced by intramuscular administration of $10 \mathrm{mg} / \mathrm{kg}$ of Zoletil and $0.05 \mathrm{mg} / \mathrm{Kg}$ of Atropine and maintained with gas (isofluorane, nitrous oxide, and oxygen) together with $5 \mathrm{mg} / \mathrm{kg} / \mathrm{h}$ of Propofol intravenous infusion. Animals were mechanically ventilated (respiratory volume: $150 \mathrm{~mL} / \mathrm{Kg} / \mathrm{min}$, respiratory rate: 15 cycles/min) and sacrificed by $\mathrm{KCl}$ i.v. injection under anaesthesia.

2.1.3. Tissue Processing. The femoral artery (FA) and the proximal tract of the right coronary artery (RCA) were isolated and 25-30 $\mathrm{mm}$ long segments excised and quickly placed in serum-free medium for secreted protein collection. Thereafter the entire heart was immersed in 5\% buffered formalin for tissue fixation (5-7 days) and subsequent coronary segmentation for histology and immunohistochemistry.

2.2. Secreted Protein Collection Procedure. Immediately after heart arrest, RCA and FA segments from CTRL and HF cases were processed according to literature and collected proteins were analysed by HPLC-MS/MS analysis [18]. Briefly, samples were incubated in 6-well plates in $2 \mathrm{~mL}$ of Eagle's Minimal Essential Medium (Sigma-Aldrich, USA) supplemented with Penicillin and Streptomycin, without Fetal Bovine Serum (FBS) and Phenol Red at $37^{\circ} \mathrm{C}$ in a humidified atmosphere of $5 \% \mathrm{CO}_{2}$. After three hours, the medium was replaced. After $24 \mathrm{~h}$, the culture medium was harvested, centrifuged at $300 \times \mathrm{g}$ for $10 \mathrm{~min}$. Samples were concentrated by centrifugal 
devices Amicon Ultra-3 (Millipore, Germany) following the manufacturer's recommendations.

2.3. Reduction, Alkylation, and Digestion of Proteins. Each secretome sample was processed, by preparing a solution of $1 \mu \mathrm{g} / \mu \mathrm{L}$ of proteins and $40 \mathrm{mM}$ of ammonium hydrogen carbonate (Sigma-Aldrich, USA). Reduction was obtained by adding $5 \mathrm{mM}$ dithiothreitol to each sample, with an incubation of $20 \mathrm{~min}$ at $80^{\circ} \mathrm{C}$. Finally alkylation was obtained by adding $10 \mathrm{mM}$ iodoacetamide and incubation for $30 \mathrm{~min}$ at $37^{\circ} \mathrm{C}$. Digestion was performed incubating the samples overnight with $0.25 \mathrm{mg} / \mathrm{mL}$ of trypsin solution at $37^{\circ} \mathrm{C}$ (substrate/enzyme $=100 / 1$ ).

2.4. LC-MS/MS Analysis and Data Processing. Chromatographic separation of digested peptides was performed using an Ultimate 3000 nano-HPLC system (LC Packings, DIONEX, USA) and peptides eluted from chromatographic C18 column were directly processed using TripleTOF 5600 mass spectrometer (ABSCIEX, Canada). For each secretome sample (CTRL $N=6$, HF FA $N=6$ and HF RCA $N=6$ ), two technical replicates were injected.

The mass spectrometer was controlled by Analyst 1.6.1 software (AB SCIEX, Toronto, Canada). For positive ionization, ion source parameters were the following: spray voltage was $3 \mathrm{kV}$ and source temperature $150^{\circ} \mathrm{C}$ with curtain gas set at 25, GS1 10, and GS2 0 psi nitrogen flow. For information dependent acquisition (IDA) analysis, survey scans were acquired in $250 \mathrm{~ms}$ and 25 product ion scans were collected if exceeding a threshold of 125 counts per second (counts/s). The total cycle time was fixed to $1.25 \mathrm{~s}$. Four time bins were added for each scan at a pulser frequency value of $11 \mathrm{kHz}$ through monitoring of the $40 \mathrm{GHz}$ multichannel TDC detector with four-anode/channel detection. Dynamic exclusion was set to $1 / 2$ of peak width $(\sim 8 s)$, and then the precursor was refreshed off the exclusion list.

MS/MS data were processed with ProteinPilot Software (ABSCIEX, Canada), using the Paragon and Pro Group Algorithms and SwissProt 2013 as protein database for Sus scrofa. The false discovery rate (FDR) analysis was performed using the integrated tools in ProteinPilot software and a confidence level of $95 \%$ was set to exclude false positive sequence identifications by analyzing the specificity and the quality of results with decoy database searching. Expression data for proteins and label-free comparative analysis were obtained using MarkerView software 1.2.1 (ABSCIEX, Canada).

2.5. Western Blot Analysis of Secretome Samples. The same secretome samples used for HPLC-MS analysis (CTRL, HF) were tested on a $10 \%$ SDS-PAGE; separated proteins transferred onto a nitrocellulose membrane (Amersham, GE Healthcare, USA) using a wet transfer system (Bio-Rad Laboratories, USA). Membranes were blocked with 3\% BSA in TBST for $1 \mathrm{~h}$ at room temperature. Primary and secondary antibodies were diluted in 3\% BSA in TBST. All primary antibodies were incubated overnight at $4^{\circ} \mathrm{C}$. HRP-conjugated secondary antibodies (Santa Cruz Biotechnology, USA) were incubated for $1 \mathrm{~h}$ at room temperature.
The following antibodies were used: CATD (C20), goat polyclonal (Santa Cruz Biotechnology, USA), dilution 1:300, and CH3L1 goat polyclonal (R\&D Systems), dilution 1:500.

Densitometric quantification of photographic films was performed using Quantity One 1-D Analysis Software (BioRad Laboratories, USA). Photographic films were scanned and analysed by Quantity One 1-D Analysis Software (BioRad Laboratories, USA).

2.6. Histology and Immunohistochemistry. After incubation for secretome analysis, all segments of RCA and FA were placed in 5\% buffered formalin and processed for paraffin embedding: longitudinal $5 \mu \mathrm{m}$ thick consecutive sections were obtained by a rotary microtome (Microm HM 300, Biooptica) and stained with Haematoxylin and Eosin (H\&E) and Masson's trichrome stain in order to observe and morphometrically assess pathologic changes. The same procedure and analysis were carried out on 2 additional crosssectioned segments of RCA adjacent to those sampled for secretome analysis (distal and proximal side) in each case; other coronary samples were harvested from left main $(n=$ $2)$, left anterior descending $(n=10)$, and left circumflex arteries $(n=4)$ in each CTRL and HF case and processed for histology in order to morphometrically assess overall coronary ATS burden. A total of 20 to 30 consecutive serial sections were obtained from each segment for histologic staining and immunohistochemistry. For immunohistochemistry, sections were placed on positively charged slides, deparaffinized, rehydrated, and washed in distilled water. After incubation in $\mathrm{H}_{2} \mathrm{O}_{2}$ at room temperature, antigen retrieval was accomplished (citrate buffer $\mathrm{pH}=6$ in microwave for $10 \mathrm{~min}$ at $500 \mathrm{~W}$ ) and then sections incubated with diluted normal blocking serum. The following primary antibodies were used: anti- $\alpha \mathrm{SM}$-actin (alpha smooth muscle actin, clone 1A4 ADB, Serotec) as a VSMC phenotype marker, anti-S100 A4 (rabbit polyclonal antibody diluted 1:200, Novus Biologicals) as a marker of synthetic VSMC phenotype $[19,20]$, and anti-CHI3L1 (goat polyclonal antibody diluted $1: 40, \mathrm{R} \& \mathrm{D}$ Systems) as a marker derived from the results of secretome analysis. They were applied overnight on the slides in a $4^{\circ} \mathrm{C}$ humid chamber. Following 30 min biotinylated secondary antibody and 30 min Vectastain Elite $A B C$ reagent incubation in Peroxidase substrate solution (DAB), slides were counterstained with Mayer's Haematoxylin for $1 \mathrm{~min}$ and mounted (Neo-Entellan Merk). Omission of the primary antibody served as a negative control. Antibody binding is visible as brown or dark brown stain (DAB); negative cells are stained blue (Haematoxylin counterstain).

\subsection{Morphometry and Quantitative Immunohistochemistry.} Consecutive longitudinal and cross sections of each arterial sample were examined under a light microscope (Olympus $\mathrm{BX} 43)$ at $4 \mathrm{x}$ to $40 \mathrm{x}$ original magnification and digitized by a video system (Olympus D20 camera) interfaced to Olympus Cell Sens Dimension software for image acquisition and analysis.

Arterial wall changes were analysed by two independent pathologists who attempted to relate them to the current 
histological grading according to American Heart Association (AHA) classification guidelines [8, 21].

The following morphometric indexes were calculated by mean value of 5 consecutive cross sections of each identified lesion at the site of its maximal extent: maximal intimal thickness (IT), maximal intimal to media thickness ratio (IMT ratio), cross-sectional lesion area (LA), and lesion to intact wall area ratio (LA ratio).

All RCA segments used for secretome analysis were morphometrically analysed; additional RCA, left main, left anterior descending, and left circumflex artery segments were also analysed to estimate overall coronary ATS burden from morphometry in each case of HF group. Results were correlated with those of corresponding coronary samples processed for secretome analysis. A total number of 134 coronary segments were morphometrically analysed (see Table S4 in Supplementary Material available online at http://dx.doi.org/10.1155/ 2015/465242).

Quantitative analysis of antibody staining of full lesion area was carried out by averaging microscopic measurements of 3 consecutive sections of each segment, digitized at $20 \mathrm{x}$ magnification under the same light source settings, and processed by semiautomatic color thresholding of Olympus Cell Sens Dimension software: positive-staining area was expressed as percentage of dark brown pixels of the entire lesion area and used as a comprehensive index of antibody binding.

Tissue codistribution and cell colocalization of different antibodies in the same region and cell type, respectively, were assessed by comparing them with positive tissue/cell in the corresponding microscopic field of adjacent consecutive sections.

2.8. ELISA of Plasma Samples. Dosage by double-antibody sandwich enzyme-linked immunosorbent assay (ELISA) was performed for CHI3L1. ELISA kit was used and reagents were prepared following the manufacturer's manual of Uscn Life Science Inc. All standards and plasma samples collected before surgery from the same animals (CTRL $N=6$ and HF $N=6$ ) were assayed in duplicate. The OD absorbance at $450 \mathrm{~nm}$ was read by a FLUOstar Omega microplate reader (BMG Labtechmicroplate).

2.9. Data Analysis. To evaluate differentially released proteins by CTRL, HF RCA, and HF FA segments (the latter for internal control), MarkerView 1.2 software was used and principal component analysis (PCA) was performed on mass spectrometric data of individually different biological samples to assess global differences between the groups (Figure S5 in Supplementary Material). Comparative analysis in MarkerView was performed, comparing MS area of the peptide eluted peaks with total area of the peptide peaks for proteins. Normalization was accomplished by LC-MS profiles (normalization based on total MS derived total ioncurrent area as an estimate of total protein content). Two technical replicates for biological sample were used and mean values were obtained for comparative analysis (Table S3 in Supplementary Material). Principal component analysis (PCA) was performed in order to identify groups within the dataset. The three groups (CTRL, HF FA, and HF RCA) were compared by $t$-test statistics: CTRL versus HF FA, CTRL versus HF RCA, and HF FA versus HF RCA samples.

Paired $t$-test was used as statistical parameter between the means of continuous variables to determine significant differences between the two categories of mass spectrometric data. Fold change $>2$ and $P$ value $<0.05$ were considered significant to validate differences between categories.

Statistical analyses of other data were conducted using Origin 7.0 software (Origin Lab, USA). Data were expressed as the mean \pm SD. Differences between the means of the 2 continuous variables were evaluated by Student's $t$-test and results were accepted when $t$-test $>95 \%(P$ value $<0.05)$. Paired $t$-test was used for quantitative immunohistochemistry. Ingenuity pathway analysis (IPA, http://www.ingenuity.com/products/pathways_analysis.html) was performed on a restricted number of VSMC-related proteins $(n=31)$, selected by their best correlation with at least one morphometric index of lesion extent. The subset of chosen proteins constituted of smooth muscle cell synthesized proteins and their role in cell migration and proliferation and in vascular disease was evidenced.

\section{Results}

3.1. Histology and Histomorphometry. Vessel segments used for secretome analysis and adjacent segments were histologically characterized. RCA and FA of CTRL group displayed an intact intima. Less than $2 \%$ of examined coronary segments showed small myointimal cushions at branching points as previously described in pigs on standard diet [22]. FA segments of HF group evidenced intact intima in 4 cases and Stary type I lesions in 2 cases.

Conversely, RCA from HF pigs showed atherosclerotic changes of the intima, ranging from lesions classifiable as Stary types I-III in 3 cases (preatheroma changes, pre-ATH) to those resembling types IV-V atheromas in the other 3 cases (atheroma stage, ATH).

Histologic features and average values of IMT ratio of the segments processed for secretome analysis are shown in Figure 1.

Classification of lesions according to Stary staging of ATS and average histomorphometric results of RCA and FA segments are summarized in Table 1. Average values of IT, IMT ratio, LA, and LA ratio of RCA segments of HF group displaying only preatheroma (pre-ATH) changes and those with atheroma (ATH) are reported separately; the latter subgroup shows significantly higher values.

The average values of morphometric indexes according to lesion grade in all coronary segments sampled in CTRL and HF cases are reported in Table S4 in Supplementary Material.

3.2. Proteomics Profiling of Secreted Proteins and Comparative Analysis between CTRL and HF Data. 224 proteins were identified across all samples with a Protein Score (Confidence) $>95 \%$ and local false discovery rate analysis $<1 \%$ as stringent criterion to avoid false positives (Table S1 in Supplemtary Material). Proteins were grouped using Gene Ontology (http://www.geneontology.org) and divided in exocytosis 


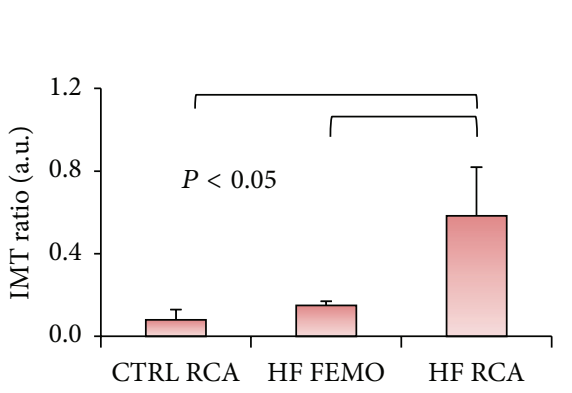

(a)

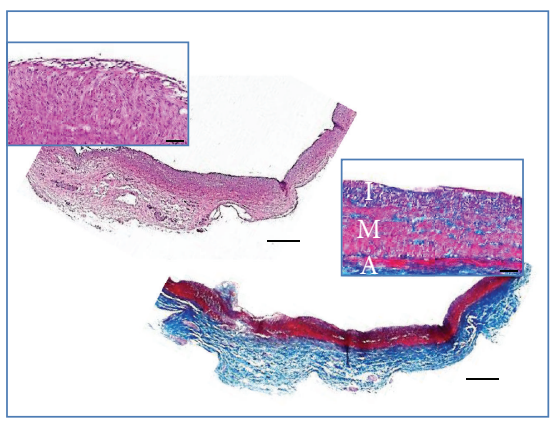

(d)

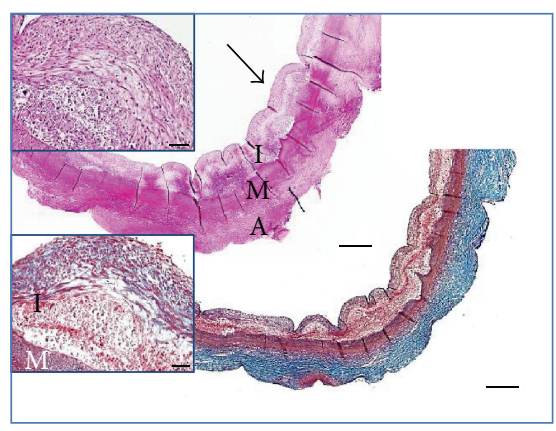

(g)

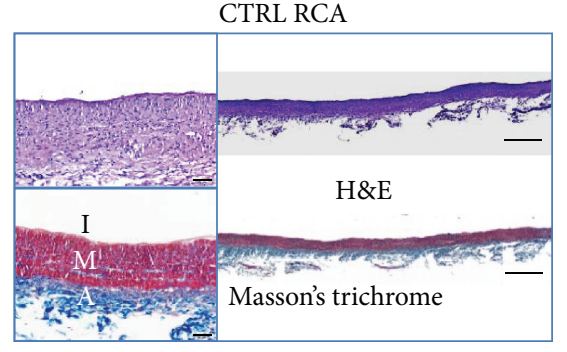

(b)

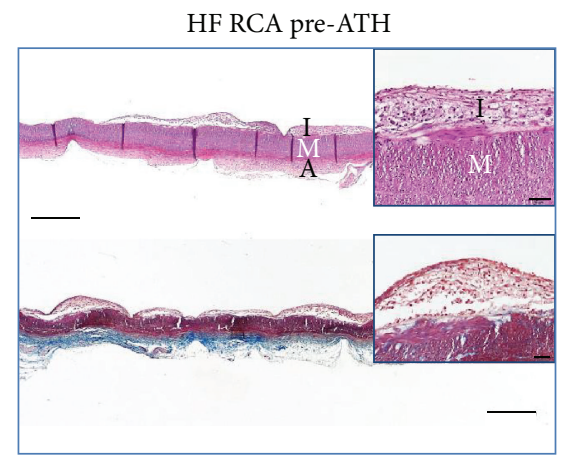

(e)

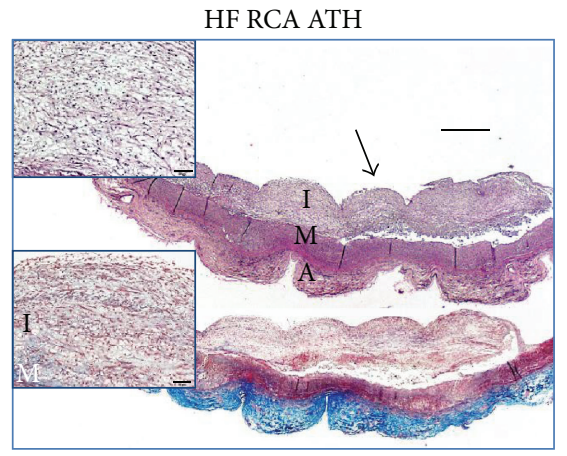

(h)

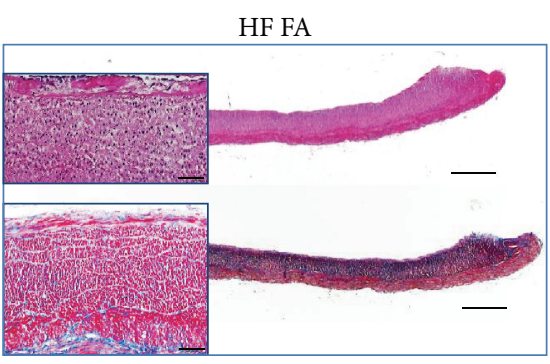

(c)

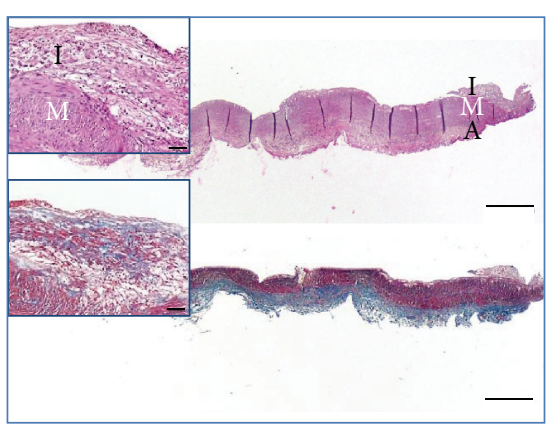

(f)

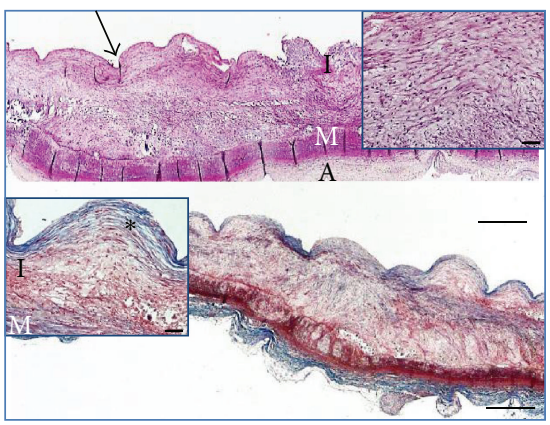

(i)

FIGURE 1: Morphometric results (panel (a)) and histologic characterization (panels (b)-(i)) of arterial segments used for secretome analysis. Panel (a): average values \pm SD of intima to media ratio (IMT) at maximal intimal thickening site in CTRL RCA, HF FA, and HF RCA segments used for secretome analysis $(n=6$ each). $P<0.05$ HF RCA versus all other segments. Panels (b)-(i): representative photomicrographs of $\mathrm{H} \& \mathrm{E}$ and Masson's trichrome stained longitudinal sections of arterial segments: intact (b) or initial type I thickening (c) in typical CTRL RCA and HF FA segments, respectively; HF RCA segments showed preatherosclerotic lesions (HF RCA pre-ATH) classifiable as Stary type I lesion (d), type II fatty streak (e), and type III lesion (f), or atheromas (black arrows, HF RCA ATH segments) classifiable as type IV ((g) and $(\mathrm{h})$ ) and as type $\mathrm{V}$ fibroatheroma ((i), fibrous cap: asterisk). Low power micrographs, bar $=500 \mu \mathrm{m}$, high power insets, bar $=50 \mu \mathrm{m}$. $\mathrm{I}=$ intima, $\mathrm{M}=$ media, $\mathrm{A}$ = adventitia.

TABLE 1: Histomorphometric results of RCA and FA segments in 6 CTRL and 6 HF cases.

\begin{tabular}{lccccc}
\hline & CTRL RCA (6) & HF FA (6) & HF RCA (6) & RCA pre-ATH (3) & RCA ATH (3) \\
\hline N/N tot. Stary types I-III & $0 / 6$ & $2 / 6$ & $3 / 6$ & $\mathbf{3} / \mathbf{3}$ & $\mathbf{0} / \mathbf{3}$ \\
N/N tot. Stary types IV-V & $0 / 6$ & $0 / 6$ & $3 / 6$ & $\mathbf{0}$ & $\mathbf{3} / \mathbf{3} \pm \mathbf{0 . 0 5}$ \\
IT (mm) & $0.03 \pm 0.02$ & $0.06 \pm 0.05$ & $0.50 \pm 0.44$ & $\mathbf{0 . 8 6} \pm \mathbf{0 . 3 6}$ \\
IMT ratio (a.u.) & $0.11 \pm 0.04$ & $0.15 \pm 0.05$ & $0.58 \pm 0.24$ & $\mathbf{0 . 3 2} \pm \mathbf{0 . 1 1}$ & $\mathbf{0 . 7 7} \pm \mathbf{0 . 0 7}$ \\
LA (mm ${ }^{2}$ ) & $0.04 \pm 0.02$ & $0.06 \pm 0.04$ & $2.61 \pm 3.42$ & $\mathbf{0 . 3 0} \pm \mathbf{0 . 2 7}$ & $\mathbf{5 . 0 0} \pm \mathbf{3 . 6 4}$ \\
LA ratio (a.u.) & $0.03 \pm 0.02$ & $0.05 \pm 0.02$ & $0.97 \pm 1.13$ & $\mathbf{0 . 1 4} \pm \mathbf{0 . 1 0}$ & $\mathbf{1 . 7 5} \pm \mathbf{1 . 1 0}$ \\
\hline
\end{tabular}

Number of segments $(N)$ and average value \pm SD of maximal IT (IT), maximal intima to media thickness ratio (IMT ratio), lesion area (LA), and lesion to intact wall area ratio of FA and RCA in CTRL and HF cases are reported. RCA segments showing Stary types I-III (RCA pre-ATH) and those displaying Stary types IV-V lesions (RCA ATH) are also grouped separately. 


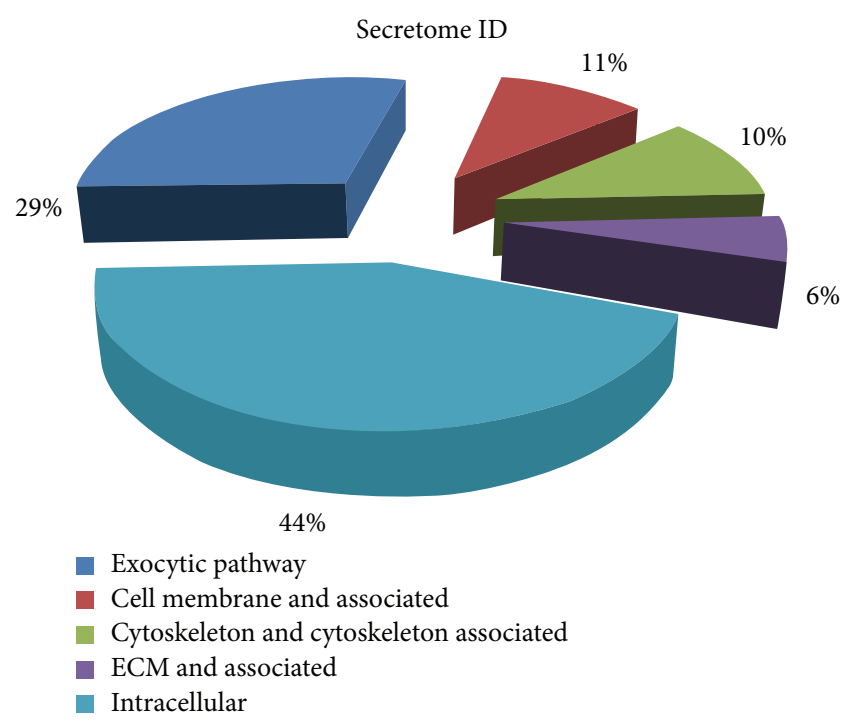

(a)

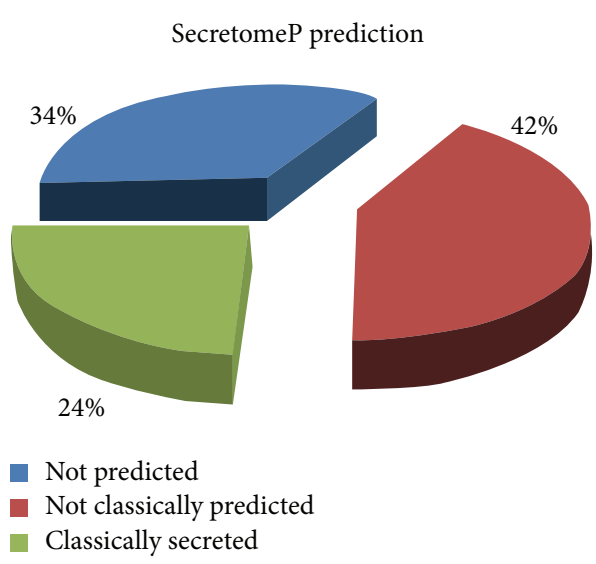

(b)

Figure 2: Pie charts of the total identified proteins from CTRL and HF. (a) Identified proteins are classified, based on Gene Ontology, according to their localization in intracellular or extracellular space. (b) Identified proteins are evaluated with SecretomeP software to compute their secretion potential. They were divided into classically secreted, not classically secreted, and not predicted.

pathway related, cell membrane and associated, cytoskeleton and associated, intracellular, and ECM associated proteins (Figure 2(a)). The secretion potential of identified proteins was computed by submitting them to SecretomeP tool which uses specific databases to predict a classical secretion via endoplasmic reticulum (presence of signal peptide) or not classical secretion through the multivesicular bodies [23] (Figure 2(b)).

PCA unsupervised clusters (Figure S5 in Supplementary Material) were CTRL samples $(N=6)$, HF FA $(N=6)$, and HF RCA $(N=6)$, which resulted in two subgroups (preATH and ATH, $N=3$ each) histologically corresponding to different ATS stage, as reported in Table 1.

Paired $t$-test evidenced 17 differentially secreted proteins when all HF RCA samples were compared to CTRL. HF FA samples were used only to distinguish between diet-related and ATS-related tissue-secreted proteins. $t$-test between HF FA and CTRL groups, which was used to suggest diet-related factors, showed significant differential expression in glyceraldehyde 3 phosphate dehydrogenase, desmin, prelamin A/C, glutathione peroxidase 1, and apolipoproteins A I and A IV.

Differentially expressed proteins were grouped into cellular $(N=11)$ and extracellular matrix proteins $(N=6)$ (Table 2). The putative roles of each factor in atherogenesis and in disease-related cellular pathways, as suggested by the literature, are reported in Table 2.

Ten out of these proteins had already been reported in serum/plasma and listed in Human Protein Reference database (http://www.hprd.org) and 15 resulted as predicted to be secreted by SecretomeP software. Western blot analysis was applied to two identified markers to validate the expression of intact proteins by an antibody-based approach and observed by mass spectrometric analysis using expression of digested peptides. Additionally, by comparing pre-ATH $(N=3)$ and ATH $(N=3)$ segments (see Figure S2 in Supplementary Material), a statistical significant difference was observed only for CHI3L1 $(P<0.05)$, which resulted as upregulated in ATH group.

The expression trends in CTRL and HF secretome samples of CHI3L1 and Cat-D were validated by Western blot, confirming the results obtained by mass spectrometry (Figure S1 in Supplementary Material).

3.3. Pathway Analysis and Tissue Immunohistochemistry. IPA was used to highlight pathways and diseases which involve secreted proteins. The most represented pathways were those related to cell proliferation, migration, and VSMC activation. Among the proteins that are implicated in atherogenesis and vascular diseases, CHI3L1 was the only one that is predicted as linked to all pathways (Figure 3).

On the basis of these results, anti-CHI3L1 antibody was used to quantify tissue expression of this protein and associate it with localization of other lesional VSMC markers and with ATS grade and morphometric indexes of lesion extent (Figure 4).

Arterial tissue immunoreactivity to anti-CHI3L1 antibody was quantified as percentage of intralesional positivestaining area in all RCA segments of CTRL and HF cases.

Anti-CHI3L1, anti- $\alpha$ SM-actin, and anti-S100A4 antibodies labelled the same region in all lesion types observed in adjacent consecutive HF RCA sections, demonstrating a consistent tissue codistribution. Colocalization of the three antibodies in the same cell type was also observed in several microscopic fields of HF RCA ATH cases (Figure 5).

ELISA for CHI3L1 detection was performed on enddiet plasma samples of the same CTRL and HF animals 
TABLE 2: Differential protein expression between HF RCA and CTRL cases.

\begin{tabular}{|c|c|c|c|c|c|c|c|}
\hline Localization & Gene name & Protein name & Coronary HF/CTRL & $P$ value & SecretomeP & Plasma & Role in atherogenesis \\
\hline \multirow{11}{*}{ Cellular } & G3P & $\begin{array}{l}\text { Glyceraldehyde-3- } \\
\text { phosphate } \\
\text { dehydrogenase }\end{array}$ & Up & 0.05 & $\sqrt{ }$ & $\sqrt{ }$ & Glucose metabolism \\
\hline & CATD & Cathepsin D & Up & 0.009 & $\sqrt{ }$ & $\sqrt{ }$ & $\begin{array}{l}\text { Macrophage phagocytosis, } \\
\text { lipid efflux }[40,41]\end{array}$ \\
\hline & DESM & Desmin & Up & 0.04 & $\sqrt{ }$ & & Focal adhesion [29] \\
\hline & VIME & Vimentin & Up & 0.02 & $\sqrt{ }$ & & Focal adhesion [29] \\
\hline & CPNS1 & Calpain & Up & 0.05 & $\sqrt{ }$ & & $\begin{array}{l}\text { Calcium binding, } \\
\text { proatherogenic [42] }\end{array}$ \\
\hline & ICAL & Calpastatin & Up & 0.05 & Not predicted & & $\begin{array}{l}\text { Calcium binding, calpain } \\
\text { inhibitor }\end{array}$ \\
\hline & MOES & Moesin & Up & 0.005 & $\sqrt{ }$ & & Focal adhesion [43] \\
\hline & CH3L1 & $\begin{array}{l}\text { Chitinase-3-like } \\
\text { protein } 1\end{array}$ & Up & 0.004 & $\sqrt{ }$ & $\sqrt{ }$ & $\begin{array}{l}\text { Adhesion and migration } \\
\text { [37] }\end{array}$ \\
\hline & S10A6 & Protein S100-A6 & Up & 0.05 & $\sqrt{ }$ & $\sqrt{ }$ & $\begin{array}{l}\text { RAGE ligand and marker of } \\
\text { MI [44] }\end{array}$ \\
\hline & LMNA & Prelamin-A/C & Up & 0.03 & Not predicted & & $\begin{array}{l}\text { Oxidative stress, vascular } \\
\text { aging [45] }\end{array}$ \\
\hline & GPX1 & $\begin{array}{l}\text { Glutathione } \\
\text { peroxidase } 1\end{array}$ & Up & 0.05 & $\sqrt{ }$ & & Oxidative stress \\
\hline \multirow{6}{*}{$\begin{array}{l}\text { Extracellular } \\
\text { matrix }\end{array}$} & PGS1 & Biglycan & $\mathrm{Up}$ & 0.04 & $\sqrt{ }$ & $\sqrt{ }$ & Lipoprotein retention [46] \\
\hline & HPLN1 & $\begin{array}{l}\text { Hyaluronan and } \\
\text { proteoglycan link } \\
\text { protein } 1\end{array}$ & Down & 0.000033 & $\sqrt{ }$ & $\sqrt{ }$ & $\begin{array}{l}\text { Plaque stability, } \\
\text { atheroprotection [47] }\end{array}$ \\
\hline & OSTP & Osteopontin & Up & 0.04 & $\sqrt{ }$ & $\sqrt{ }$ & $\begin{array}{l}\text { Secondary carotid events } \\
\text { marker [39] }\end{array}$ \\
\hline & APOA1 & Apolipoprotein A-I & Up & 0.01 & $\sqrt{ }$ & $\sqrt{ }$ & $\begin{array}{l}\text { HDL constituent, } \\
\text { atheroprotection [48] }\end{array}$ \\
\hline & APOE & Apolipoprotein E & Up & 0.0007 & $\sqrt{ }$ & $\sqrt{ }$ & Atheroprotection [49] \\
\hline & APOA4 & $\begin{array}{l}\text { Apolipoprotein } \\
\text { A-IV }\end{array}$ & Down & 0.03 & $\sqrt{ }$ & $\sqrt{ }$ & $\begin{array}{l}\text { Antioxidative, } \\
\text { atheroprotection [50] }\end{array}$ \\
\hline
\end{tabular}

First column: biological localization of proteins. Second column: gene name. Third column: protein names reported according to SwissProt 2013 database. Sixth column: secretion potential as predicted according to SecretomeP software. Seventh column: presence in plasma, according to Human Protein Reference database (http://www.hprd.org). Eighth column: roles in atherogenesis, as suggested in the literature.

and the circulatory expression of the protein resulted as $2.6 \pm 0.4 \mathrm{ng} / \mathrm{mL}$ in CTRL samples (mean $\pm \mathrm{SD}$ ) and $12.4 \pm$ $3.5 \mathrm{ng} / \mathrm{mL}$ in HF samples (mean $\pm \mathrm{SD})(P$ value $=0.001)$.

\section{Discussion}

Different proteomics approaches have been used to search for biomarkers of ATS presence and severity. The majority of these studies in humans concern carotid (from surgery) and coronary arteries with overt disease and plaque complication features [24].

Body fluids, particularly blood, are the samples of choice for biomarker discovery in medicine since they can be easily and noninvasively collected. However, plasma proteomics profiling has turned out to be extremely challenging due to the wide dynamic range of proteins, the corresponding intrinsic low abundance of potential biomarkers and the huge individual heterogeneity of the samples. For these reasons, in the last years many researchers focused on the secretome analysis of cells and tissues with the expectation that identified putative biomarkers could be traced back in body fluids with more sensitive and targeted analyses [25].

Recently, we designed and assessed a gel- and label-free LC-MS/MS workflow that was used to produce a proteome profile concerning human atherosclerotic carotid plaque and secreted proteins from cultured cells $[26,27]$.

In the present work, the overall workflow was utilized to study the secretome-contained proteins of coronary arterial segments in order to characterize the early phases of plaque formation and growth, to identify molecular markers associated with pathology grade, and to evaluate their translation into the clinical field.

To this end, a swine model of coronary atherogenesis was used, and atherosclerotic changes of different stage, extent, and distribution along the three main coronaries were observed following high fat, cholesterol-enriched diet. Despite the overall variability of the lesion distribution in the coronary segments that we analyzed (Table S4 in Supplementary Material), a four-month high fat diet treatment was able to induce in all cases coronary atherosclerotic changes 


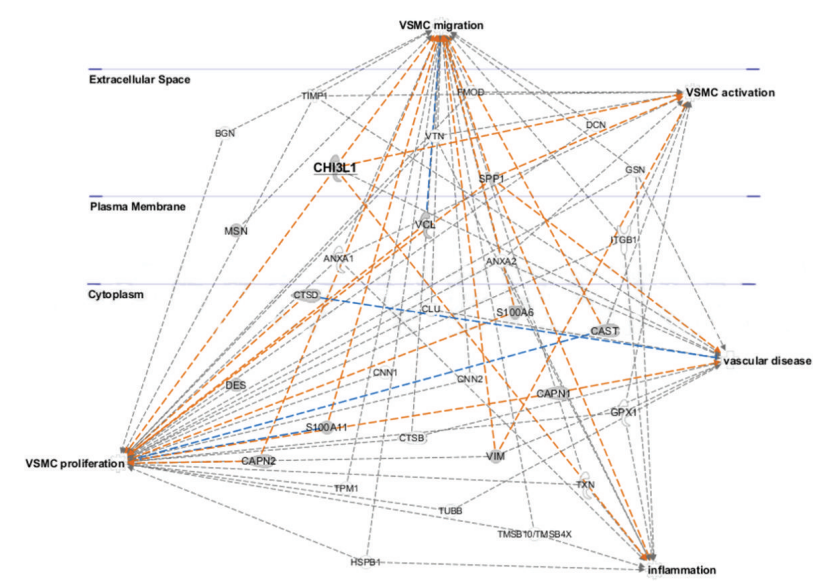

FIGURE 3: IPA of a subset of 31 proteins identified in the secretome of RCA specimens. Pathways were generated based on the information stored in IPA Knowledge base. Nodes correspond to the 31 proteins and are reported with their Gene Codes. Extracellular space, cytoplasm, and plasma membrane proteins are shown based on IPA classification. Relationships with disease and cellular functions are evidenced. The relationships that are predicted to lead to activation and to inhibition and those not predicted are reported in orange, blue, and grey, respectively.

and at least one atheroma lesion. Conversely, no changes or only type I preatherosclerotic initial changes were observed in femoral arteries, a finding supported by previous studies of porcine coronary as compared to iliac/femoral artery atherosusceptibility during diet treatment $[15,28]$. However, we cannot exclude the idea that a comprehensive histologic examination of all femoral segments, in addition to those sampled for the secretome analysis, could have evidenced more advanced atherosclerotic changes also in this artery.

LC-MS/MS analysis of the whole secretome content allowed identifying 224 proteins, among which 17 were differentially expressed between CTRL and HF cases.

t-test between HF FA and CTRL groups, both without significant atherosclerotic lesions, showed significant differential expression in glyceraldehyde 3-phosphate dehydrogenase, desmin, prelamin, glutathione peroxidase 1 , and apolipoproteins A-I and A-IV, which was attributed to events induced by different diets: an atherogenic diet-dependent modulation of lipid and glucose metabolism-associated proteins and diet induction of free radicals modulating factors related to oxidative stress. Comparison between RCA segments of CTRL and HF groups showed that the most represented differentially expressed proteins were VSMC intracellular proteins and ECM factors related to VSMC activation and synthesis.

Most of the VSMC proteins (moesin, vimentin, and desmin) that resulted in upregulation in RCA HF atherosclerotic samples, but not in HF FA samples, are related to the adhesion pathway and are strongly modulated during VSMC phenotype switch and involved in VSMC capacity to migrate [29]. This was confirmed also by bioinformatics pathway analysis (IPA software), which supported the involvement of these proteins in vascular disease and in VSMC proliferation/migration. Additionally, a relationship was found between their expression and the lesion stage and extent.

Furthermore, a significant modulation of ECM components, mostly synthesized by synthetic VSMCs, such as Biglycan, Hyaluronan, and Osteopontin, was observed in the secretome samples of HF RCA but not in HF FA samples. Proteoglycans are reported to bind cytokines and growth factors causing inflammation during ATS development [30] and may be involved in lipid retention, thus contributing to the early phases of lesion formation [31, 32]. Also many apolipoproteins (apolipoproteins A-I, A-IV, and E) are modulated in our model supporting this hypothesis.

CHI3L1 was the most relevant upregulated protein identified in HF RCA samples, which was (i) differentially expressed between CTRL, pre-ATH, and ATH samples, (ii) strongly associated with plaque size/extent and Stary stage, and (iii) immunohistologically related to a prevalent VSMC plaque composition. The site-specific association with lesion stage and size was validated for the full spectrum of diet induced lesions in samples used for secretome analysis and immunohistochemical characterization, enabled to establish the tissue and cell colocalization of CHI3L1 immunoexpression with $\alpha \mathrm{SM}$-actin/S100A 4 positive-staining areas and migratory VSMC phenotype.

At variance with previous human studies [33, 34], we did not observe the maximal anti-CHI3L1 immunoexpression in macrophages and lipid laden macrophages (Figures S2 and S3 in Supplementary Material). Conversely, maximal CHI3L1 expression was found in $\alpha \mathrm{SM}$-actin/S100A 4 positive cells, supporting its prevalent synthesis in the activated VSMC phenotype within developing atherosclerotic lesions. This difference could be explained by the more advanced stage and different cell composition of the complicated human carotid plaques as compared to coronary plaques from our experimental model of atherogenesis.

It is reported that the expression of $\mathrm{CHI} 3 \mathrm{~L} 1$ is enhanced in aorta of patients with coronary atherosclerosis and is significantly correlated with atherosclerotic risk factors [35], although increased expression of CHI3L1 in human atherosclerotic lesions is primarily associated with production and activation of inflammatory factors [36]. Despite being associated with VSMC presence in atherosclerotic plaque [37] and with VSMC activation towards a synthetic phenotype [38], this protein has never been hitherto shown to be directly correlated to coronary ATS grade and extent during atheroma formation.

CHI3L1 expression was also measured by ELISA in plasma samples of pigs fed on standard and high fat diet. The statistically significant upregulation of its circulatory expression consolidates the association between the expression of this marker and the presence of CAD in this experimental model of atherogenesis and supports its amenability to be used as marker of ATS severity in the clinical context.

Altogether, these data confirm that VSMC activation towards a migratory/synthetic phenotype may play a pivotal role in the early formation and progression of coronary 


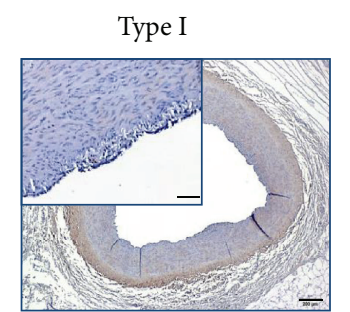

(a)
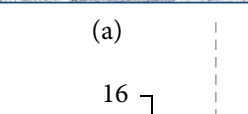

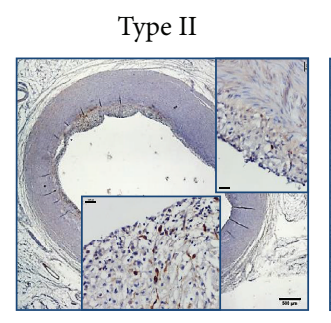

(b)

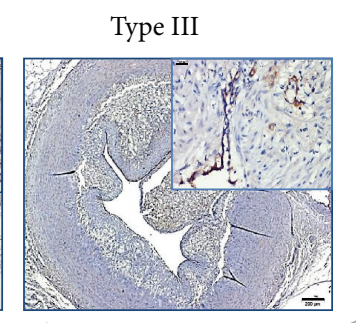

(c)
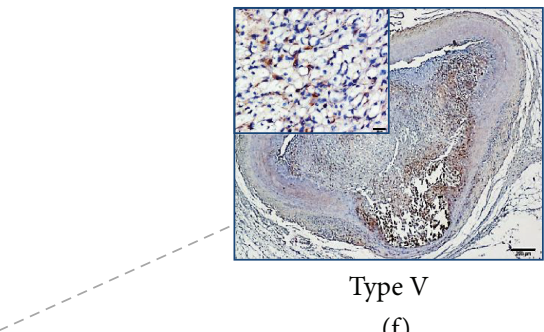

Type V

(f)

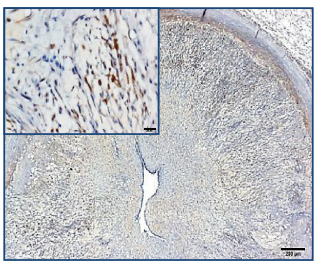

Type IV

(e)

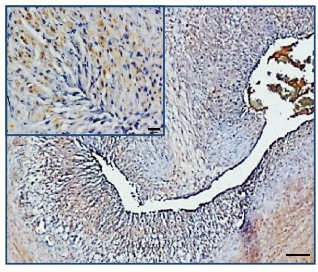

Type IV

(d)

IMT ratio (a.u.)

Secreted CHI3L1 expression (a.u.)

Lesional CHI3L1 immunoexpression (\% positive area)

FIGURE 4: Correlation between secreted and tissue coronary CHI3L1 expression (by LC-MS/MS analysis and immunohistochemistry, resp.) and IMT ratio of corresponding segments at different Stary stages. Representative photomicrographs at low (bar $=200 \mu \mathrm{m})$ and high magnification (enlargements of intimal lesion area, insets; bar $=50 \mu \mathrm{m}$ ) from CHI3L1 antibody labeled cross sections of HF RCA segments (from (a) to (f)), according to ATS grade (Stary types I to V) demonstrate the correlation of CHI3L1 expression (dark brown staining indicates positivity) and of ATS stage with secreted CHI3L1 values (arrows). Secreted CHI3L1 expression (blue dots) and CHI3L1 immunoreactivity, expressed as percentage of intralesional positive cell area (red squares), hold direct and significantly linear relations $\left(R^{2}>0.8\right)$ with the IMT ratio of the same segments.

atheromas and suggest that VSMC-derived/-secreted molecules can be usefully exploited as plaque stage/size related biomarkers in CAD.

4.1. Study Limitations. The major drawbacks in atherogenesis research are (i) difficulties in investigating the early phase of the pathology in the clinical context and (ii) discrepancies between experimental models and human characteristics of the disease, which can limit the relevance of results. The use of highly unbalanced diets and/or genetic manipulation are the only possible choices to produce appreciable atherosclerotic changes within few months as technically required. The atherogenic diet adopted in our study to induce accelerated coronary lesions in pigs could make it difficult to extrapolate results credibly from the experimental to the clinical context; however, the diet-independent relation to site-specific and feature-specific characteristics of coronary atherosclerotic plaque suggests our proteomics findings as realistically informative in the field of marker discovery for disease initiation and evolution.
This hypothesis is supported by the evidence that, despite intragroup variability of lesion grade and distribution, morphometric indexes of single RCA segments processed for secretome analysis are directly and significantly related to the average coronary values of the corresponding cases (see Figure S4 and Table S4 in Supplementary Material), thus extending the relevance of site-specific proteomics results to the entire atherosclerotic coronary tree.

Despite the limited number of samples analysed, the observation that several plaque-secreted proteins identified in our animal model have been previously found overexpressed also in human atherosclerotic tissue and blood [35, 39] indirectly reinforces the translational utility of our study.

\section{Conclusion}

The outcomes of this study are the following:

(1) The most relevant VSMC-secreted proteins are related to coronary ATS stage and extent, suggesting that 

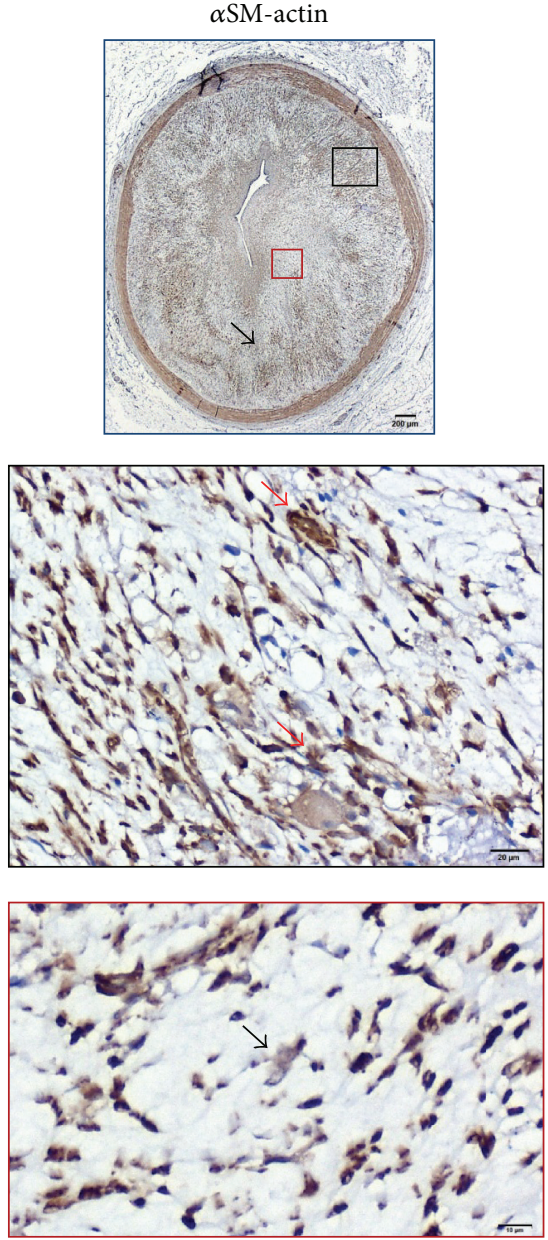

S100A4
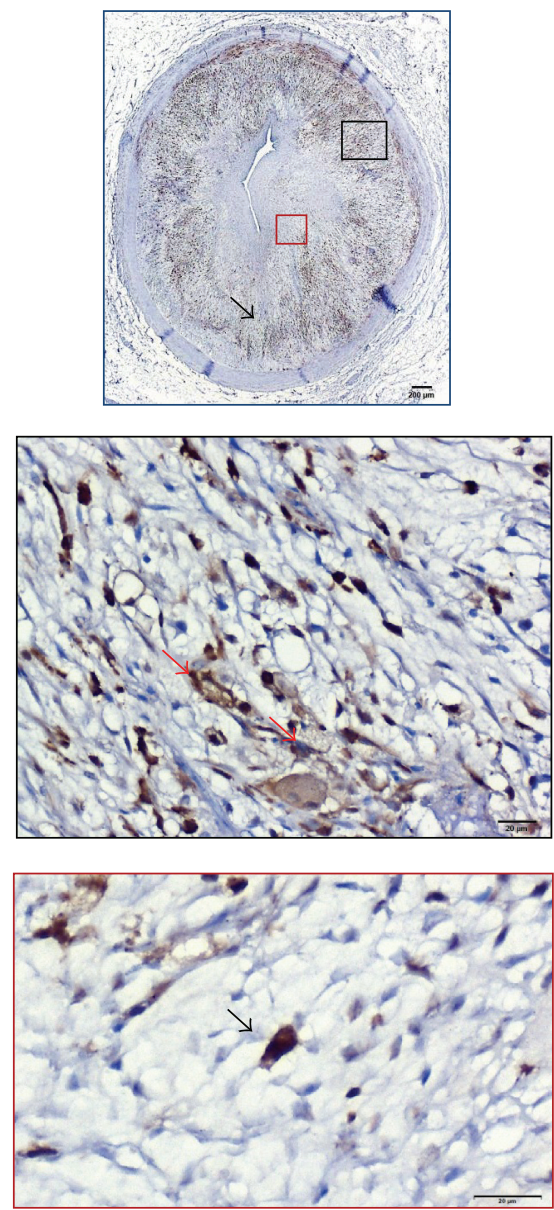

CHI3L1
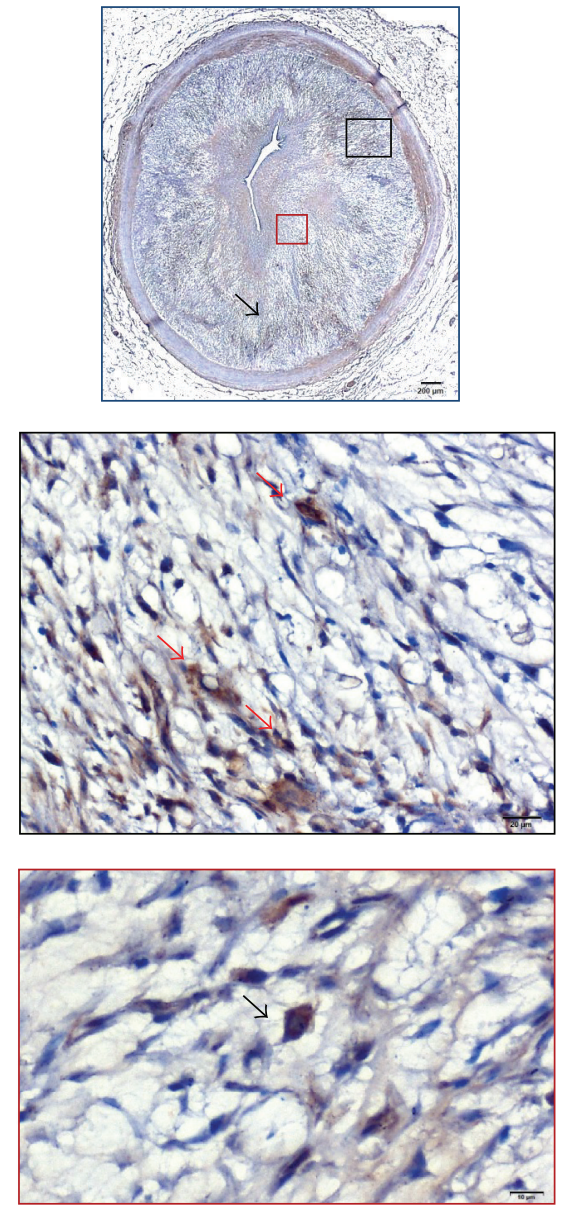

FIGURE 5: Representative photomicrographs of immunostained consecutive cross sections of RCA in a typical ATH case of HF group (immunopositive cells are dark brown). Immunostained sections demonstrate tissue codistribution (top row) and cell colocalization (middle and bottom rows) of anti-CHI3L1 with anti- $\alpha$ SM-actin and with anti-S100A4 antibodies in a fibrolipid plaque. Enlargements of plaque regions (black and red insets) are displayed in middle and bottom row, respectively. Plaque areas labelled by anti- $\alpha \mathrm{SM}$-actin, anti-S100A4, and antiCHI3L1 antibodies are indicated (top panels, black arrows; bar $=200 \mu \mathrm{m}$ ) and colocalization of all three antibodies in the same cell type evidenced (bottom panels, black arrows; bar $=10 \mu \mathrm{m}$ ). CHI3L1 immunopositive cells resulted also in $\alpha$ SM-actin or S100A4 positive or both (middle panels, red arrows; bar $=20 \mu \mathrm{m}$ ).

VSMCs regulate and contribute to the initial stages of CAD. Moreover, profiling data indicate a general reorganization of VSMC-based pathways connected with CAD severity, supporting the role of activated VSMCs in the evolution of coronary lesions from fatty streak to atheroma.

(2) CHI3L1 is the most strongly associated protein with coronary ATS grade and extent.

(3) Immunohistochemistry suggests prevalent localization of CHI3L1 in S100A4 positive VSMCs and supports the stage-specific distribution of this marker in the full spectrum of coronary lesions, from initial fatty streak to fibroatheroma.

(4) ELISA of CHI3L1 confirms a statistical significant association between its circulatory level and the presence of CAD.
This approach can help identify circulating markers of early plaque development and/or fast growth that could be validated in a large clinically characterized cohort of patients, contributing to improving risk assessment of primary coronary events in CAD.

\section{Ethical Approval}

Animal instrumentation and experimental protocols were approved by the Animal Care Committee of the Italian Ministry of Health (protocol number: 06/2009-B-2009/01/26) according with the Italian Law (DL-116, January 27, 1992) and National Institute of Health Guide for the Care and Use of Laboratory Animals.

\section{Conflict of Interests}

The authors declare that there is no conflict of interests regarding the publication of this paper. 


\section{Authors' Contribution}

Silvia Rocchiccioli and Antonella Cecchettini contribute equally to this work.

\section{Acknowledgments}

The authors would like to thank Dr. Claudia Kusmic for conducting the animal diet, Dr. Fabio Bernini, Dr. Silvia Burchielli, and Dr. Federico Vozzi for their assistance in the animal study, and Dr. Michela Rial for paper revision. This work was supported by ARTreat FP7-ICT-2008 project Multi-Level Patient-Specific Artery and Atherogenesis Model for Outcome Prediction, Decision Support Treatment, and Virtual Hand-On Training [Grant Agreement FP7 224297] and by VPH2 FP7-ICT-2007 project Virtual Pathological Heart of the Virtual Physiological Human [Grant Agreement FP7 224635].

\section{References}

[1] A. J. Lusis, "Atherosclerosis," Nature, vol. 407, no. 6801, pp. $233-$ 241, 2000.

[2] P. Libby, P. M. Ridker, and G. K. Hansson, "Progress and challenges in translating the biology of atherosclerosis," Nature, vol. 473, no. 7347, pp. 317-325, 2011.

[3] A. V. G. Edwards, M. Y. White, and S. J. Cordwell, "The role of proteomics in clinical cardiovascular biomarker discovery," Molecular and Cellular Proteomics, vol. 7, no. 10, pp. 1824-1837, 2008.

[4] M. C. Duran, S. Mas, J. L. Martin-Ventura et al., "Proteomic analysis of human vessels: application to atherosclerotic plaques," Proteomics, vol. 3, no. 6, pp. 973-978, 2003.

[5] F. de la Cuesta, I. Zubiri, A. S. Maroto et al., "Deregulation of smooth muscle cell cytoskeleton within the human atherosclerotic coronary media layer," Journal of Proteomics, vol. 82, pp. 155-165, 2013.

[6] F. de la Cuesta, M. G. Barderas, E. Calvo et al., "Secretome analysis of atherosclerotic and non-atherosclerotic arteries reveals dynamic extracellular remodeling during pathogenesis," Journal of Proteomics, vol. 75, no. 10, pp. 2960-2971, 2012.

[7] M. R. Alexander and G. K. Owens, "Epigenetic control of smooth muscle cell differentiation and phenotypic switching in vascular development and disease," Annual Review of Physiology, vol. 74, pp. 13-40, 2012.

[8] C. Chaabane, C. W. Heizmann, and M.-L. Bochaton-Piallat, "Extracellular S100A4 induces smooth muscle cell phenotypic transition mediated by RAGE," Biochimica et Biophysica Acta, vol. 1853, no. 9, pp. 2144-2157, 2015.

[9] H. C. McGill, C. A. McMahan, and S. S. Gidding, "Preventing heart disease in the 21st century implications of the pathobiological determinants of atherosclerosis in youth (PDAY) study," Circulation, vol. 117, no. 9, pp. 1216-1227, 2008.

[10] F. Diet, R. E. Pratt, G. J. Berry, N. Momose, G. H. Gibbons, and V. J. Dzau, "Increased accumulation of tissue ACE in human atherosclerotic coronary artery disease," Circulation, vol. 94, no. 11, pp. 2756-2767, 1996.

[11] Y. Nakashima, T. N. Wight, and K. Sueishi, "Early atherosclerosis in humans: role of diffuse intimal thickening and extracellular matrix proteoglycans," Cardiovascular Research, vol. 79, no. 1, pp. 14-23, 2008.
[12] E. Hai, Y. Ikura, T. Naruko et al., "Alterations of endothelinconverting enzyme expression in early and advanced stages of human coronary atherosclerosis," International Journal of Molecular Medicine, vol. 13, no. 5, pp. 649-654, 2004.

[13] Y. Nakashima, H. Fujii, S. Sumiyoshi, T. N. Wight, and K. Sueishi, "Early human atherosclerosis: accumulation of lipid and proteoglycans in intimal thickenings followed by macrophage infiltration," Arteriosclerosis, Thrombosis, and Vascular Biology, vol. 27, no. 5, pp. 1159-1165, 2007.

[14] D. Hamamdzic and R. L. Wilensky, "Porcine models of accelerated coronary atherosclerosis: role of diabetes mellitus and hypercholesterolemia," Journal of Diabetes Research, vol. 2013, Article ID 761415, 7 pages, 2013.

[15] E. R. Mohler III, L. Sarov-Blat, Y. Shi et al., "Site-specific atherogenic gene expression correlates with subsequent variable lesion development in coronary and peripheral vasculature," Arteriosclerosis, Thrombosis, and Vascular Biology, vol. 28, no. 5, pp. 850-855, 2008.

[16] N. Dejeans, S. Auclair, S. Chauvet, D. Milenkovic, and A. Mazur, "Transcriptomic analysis of aorta from a short-term high-fat diet fed mouse reveals changes in the expression of vessel structure genes," Journal of Physiology and Pharmacology, vol. 60, supplement 1, pp. 37-45, 2009.

[17] W. T. Friedewald, R. I. Levy, and D. S. Fredrickson, "Estimation of the concentration of low-density lipoprotein cholesterol in plasma, without use of the preparative ultracentrifuge," Clinical Chemistry, vol. 18, no. 6, pp. 499-502, 1972.

[18] S. Rocchiccioli, G. Pelosi, S. Rosini et al., "Secreted proteins from carotid endarterectomy: an untargeted approach to disclose molecular clues of plaque progression," Journal of Translational Medicine, vol. 11, no. 1, article 260, 2013.

[19] A. C. Brisset, H. Hao, E. Camenzind et al., "Intimal smooth muscle cells of porcine and human coronary artery express S100A4, a marker of the rhomboid phenotype in vitro," Circulation Research, vol. 100, no. 7, pp. 1055-1062, 2007.

[20] H. C. Stary, A. B. Chandler, S. Glagov et al., "A definition of initial, fatty streak, and intermediate lesions of atherosclerosis: a report from the committee on vascular lesions of the council on arteriosclerosis, American Heart Association," Circulation, vol. 89, no. 5, pp. 2462-2478, 1994.

[21] H. C. Stary, "Natural history and histological classification of atherosclerotic lesions an update," Arteriosclerosis, Thrombosis, and Vascular Biology, vol. 20, no. 5, pp. 1177-1178, 2000.

[22] V. Fuster, J. T. Lie, L. Badimon, J. A. Rosemark, and E. J. Bowie, "Spontaneous and diet-induced coronary atherosclerosis in normal swine and swine with von Willebrand disease," Arteriosclerosis, vol. 5, no. 1, pp. 67-73, 1985.

[23] J. D. Bendtsen, L. J. Jensen, N. Blom, G. Von Heijne, and S. Brunak, "Feature-based prediction of non-classical and leaderless protein secretion," Protein Engineering, Design and Selection, vol. 17, no. 4, pp. 349-356, 2004.

[24] O. B. Bleijerveld, Y.-N. Zhang, S. Beldar et al., "Proteomics of plaques and novel sources of potential biomarkers for atherosclerosis," Proteomics-Clinical Applications, vol. 7, no. 7-8, pp. 490-503, 2013.

[25] K. J. Brown, H. Seol, D. K. Pillai et al., "The human secretome atlas initiative: implications in health and disease conditions," Biochimica et Biophysica Acta (BBA)_Proteins and Proteomics, vol. 1834, no. 11, pp. 2454-2461, 2013.

[26] S. Rocchiccioli, G. Pelosi, S. Rosini et al., "Secreted proteins from carotid endarterectomy: an untargeted approach 
to disclose molecular clues of plaque progression," Journal of Translational Medicine, vol. 11, article 260, 2013.

[27] L. Comelli, S. Rocchiccioli, S. Smirni et al., "Characterization of secreted vesicles from vascular smooth muscle cells," Molecular BioSystems, vol. 10, no. 5, pp. 1146-1152, 2014.

[28] L. Trigueros-Motos, J. M. Gonzalez-Granado, C. Cheung et al., "Embryological-origin-dependent differences in homeobox expression in adult aorta: role in regional phenotypic variability and regulation of NF- $\kappa \mathrm{B}$ activity," Arteriosclerosis, Thrombosis, and Vascular Biology, vol. 33, no. 6, pp. 1248-1256, 2013.

[29] S. Rocchiccioli, N. Ucciferri, L. Comelli, M. G. Trivella, L. Citti, and A. Cecchettini, "Proteomics changes in adhesion molecules: a driving force for vascular smooth muscle cell phenotypic switch," Molecular BioSystems, vol. 8, no. 4, pp. 1052-1059, 2012.

[30] T. W. L. Groenevdd, M. Oroszlán, R. T. Owens et al., "Interactions of the extracellular matrix proteoglycans decorin and biglycan with Clq and collectins," Journal of Immunology, vol. 175, no. 7, pp. 4715-4723, 2005.

[31] V. Y. Anggraeni, N. Emoto, K. Yagi et al., "Correlation of C4ST1 and ChGn-2 expression with chondroitin sulfate chain elongation in atherosclerosis," Biochemical and Biophysical Research Communications, vol. 406, no. 1, pp. 36-41, 2011.

[32] R. Y. Rasente, P. Egitto, and G. C. Calabrese, "Low molecular mass dermatan sulfate modulates endothelial cells proliferation and migration," Carbohydrate Research, vol. 356, pp. 233-237, 2012.

[33] R. G. Boot, T. A. E. van Achterberg, B. E. van Aken et al., "Strong induction of members of the chitinase family of proteins in atherosclerosis: chitotriosidase and human cartilage gp-39 expressed in lesion macrophages," Arteriosclerosis, Thrombosis, and Vascular Biology, vol. 19, no. 3, pp. 687-694, 1999.

[34] A. E. Michelsen, C. N. Rathcke, M. Skjelland et al., "Increased YKL-40 expression in patients with carotid atherosclerosis," Atherosclerosis, vol. 211, no. 2, pp. 589-595, 2010.

[35] Z. Gong, S. Xing, F. Zheng, and Q. Xing, "Increased expression of chitinase 3-like 1 in aorta of patients with atherosclerosis and suppression of atherosclerosis in apolipoprotein e-knockout mice by chitinase 3-like 1 gene silencing," Mediators of Inflammation, vol. 2014, Article ID 905463, 12 pages, 2014.

[36] C. G. Lee, C. A. Da Silva, C. S. Dela Cruz et al., "Role of chitin and chitinase/chitinase-like proteins in inflammation, tissue remodeling, and injury," Annual Review of Physiology, vol. 73, pp. 479-501, 2011.

[37] K. C. Nishikawa and A. J. T. Millis, "gp38k (CHI3L1) is a novel adhesion and migration factor for vascular cells," Experimental Cell Research, vol. 287, no. 1, pp. 79-87, 2003.

[38] K. M. Malinda, L. Ponce, H. K. Kleinman, L. M. Shackelton, and A. J. T. Millis, "Gp38k, a protein synthesized by vascular smooth muscle cells, stimulates directional migration of human umbilical vein endothelial cells," Experimental Cell Research, vol. 250, no. 1, pp. 168-173, 1999.

[39] D. P. De Kleijn, F. L. Moll, W. E. Hellings et al., "Local atherosclerotic plaques are a source of prognostic biomarkers for adverse cardiovascular events," Arteriosclerosis, Thrombosis, and Vascular Biology, vol. 30, no. 3, pp. 612-619, 2010.

[40] S. Jormsjö, D. M. Wuttge, A. Sirsjö et al., "Differential expression of cysteine and aspartic proteases during progression of atherosclerosis in apolipoprotein E-deficient mice," The American Journal of Pathology, vol. 161, no. 3, pp. 939-945, 2002.

[41] B. Haidar, R. S. Kiss, L. Sarov-Blat et al., "Cathepsin D, a lysosomal protease, regulates ABCA1-mediated lipid efflux,"
Journal of Biological Chemistry, vol. 281, no. 52, pp. 39971-39981, 2006.

[42] T. Miyazaki, T. Koya, Y. Kigawa et al., "Calpain and atherosclerosis," Journal of Atherosclerosis and Thrombosis, vol. 20, pp. 228237, 2013.

[43] N. Baeyens, I. Latrache, X. Yerna, G. Noppe, S. Horman, and N. Morel, "Redundant control of migration and adhesion by ERM proteins in vascular smooth muscle cells," Biochemical and Biophysical Research Communications, vol. 441, no. 3, pp. 579585, 2013.

[44] X. Y. Cai, L. Lu, Y. N. Wang et al., "Association of increased S100B, S100A6 and S100P in serum levels with acute coronary syndrome and also with the severity of myocardial infarction in cardiac tissue of rat models with ischemia-reperfusion injury," Atherosclerosis, vol. 217, no. 2, pp. 536-542, 2011.

[45] C. D. Ragnauth, D. T. Warren, Y. Liu et al., "Prelamin a acts to accelerate smooth muscle cell senescence and is a novel biomarker of human vascular aging," Circulation, vol. 121, no. 20, pp. 2200-2210, 2010.

[46] K. D. O’Brien, K. Lewis, J. W. Fischer et al., "Smooth muscle cell biglycan overexpression results in increased lipoprotein retention on extracellular matrix: implications for the retention of lipoproteins in atherosclerosis," Atherosclerosis, vol. 177, no. 1, pp. 29-35, 2004.

[47] Y. Kashima, M. Takahashi, Y. Shiba et al., "Crucial role of hyaluronan in neointimal formation after vascular injury," PLoS ONE, vol. 8, no. 3, Article ID e58760, 2013.

[48] J. K. Bielicki, M. R. McCall, and T. M. Forte, "Apolipoprotein A-I promotes cholesterol release and apolipoprotein E recruitment from THP-1 macrophage-like foam cells," Journal of Lipid Research, vol. 40, no. 1, pp. 85-92, 1999.

[49] K. L. Olin, S. Potter-Perigo, P. H. R. Barrett, T. N. Wight, and A. Chait, "Biglycan, a vascular proteoglycan, binds differently to HDL2 and HDL3: role of apoE," Arteriosclerosis, Thrombosis, and Vascular Biology, vol. 21, no. 1, pp. 129-135, 2001.

[50] M. A. Ostos, M. Conconi, L. Vergnes et al., "Antioxidative and antiatherosclerotic effects of human apolipoprotein A-IV in apolipoprotein E-deficient mice," Arteriosclerosis, Thrombosis, and Vascular Biology, vol. 21, no. 6, pp. 1023-1028, 2001. 


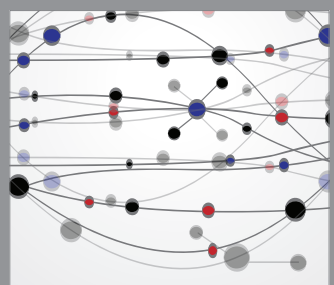

The Scientific World Journal
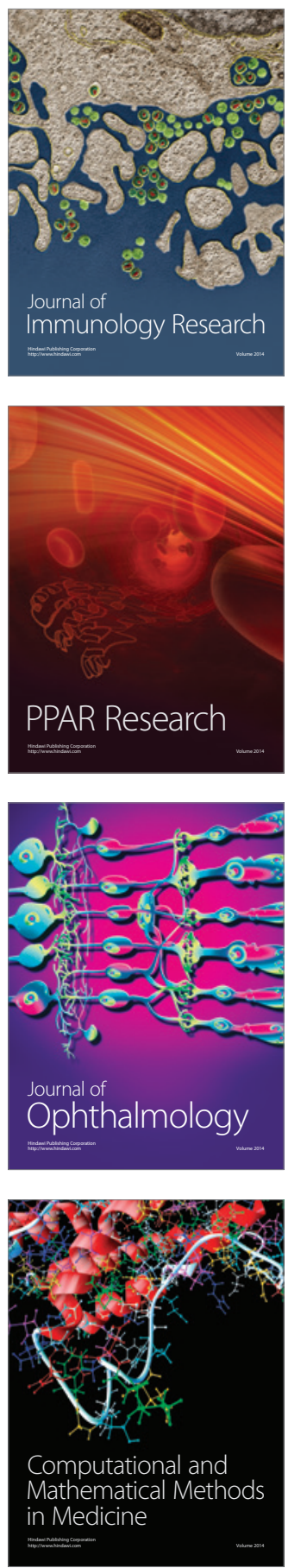

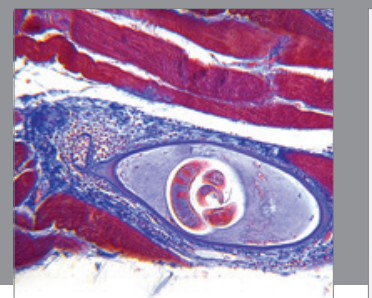

Gastroenterology

Research and Practice
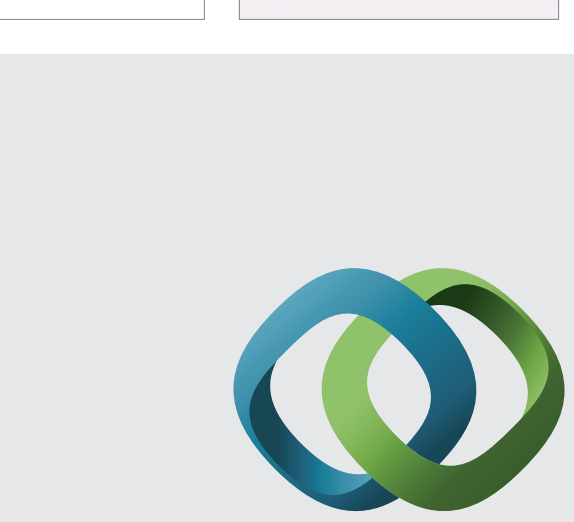

\section{Hindawi}

Submit your manuscripts at

http://www.hindawi.com
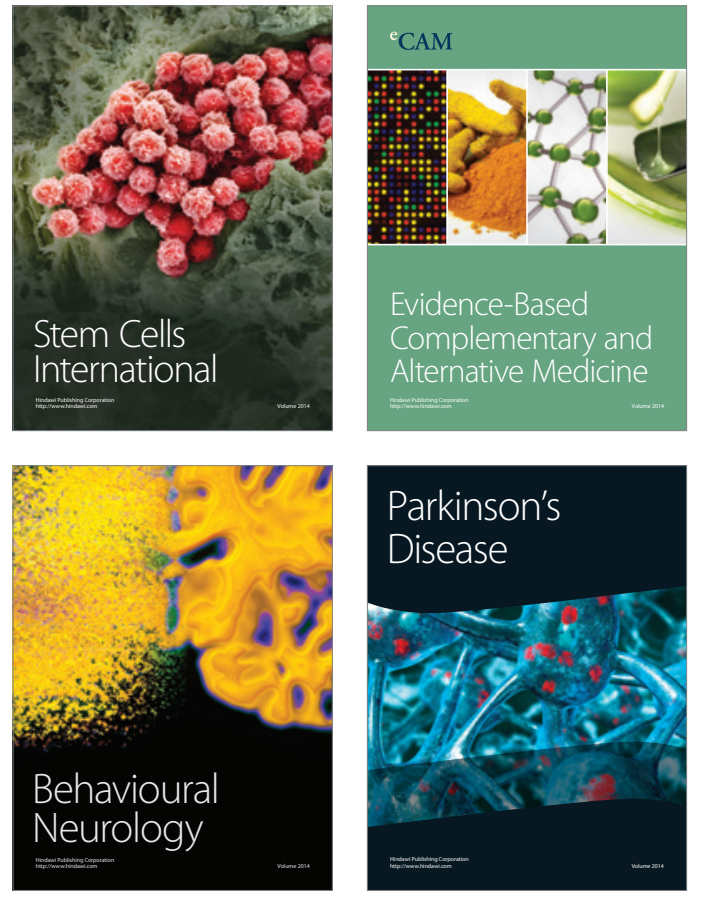
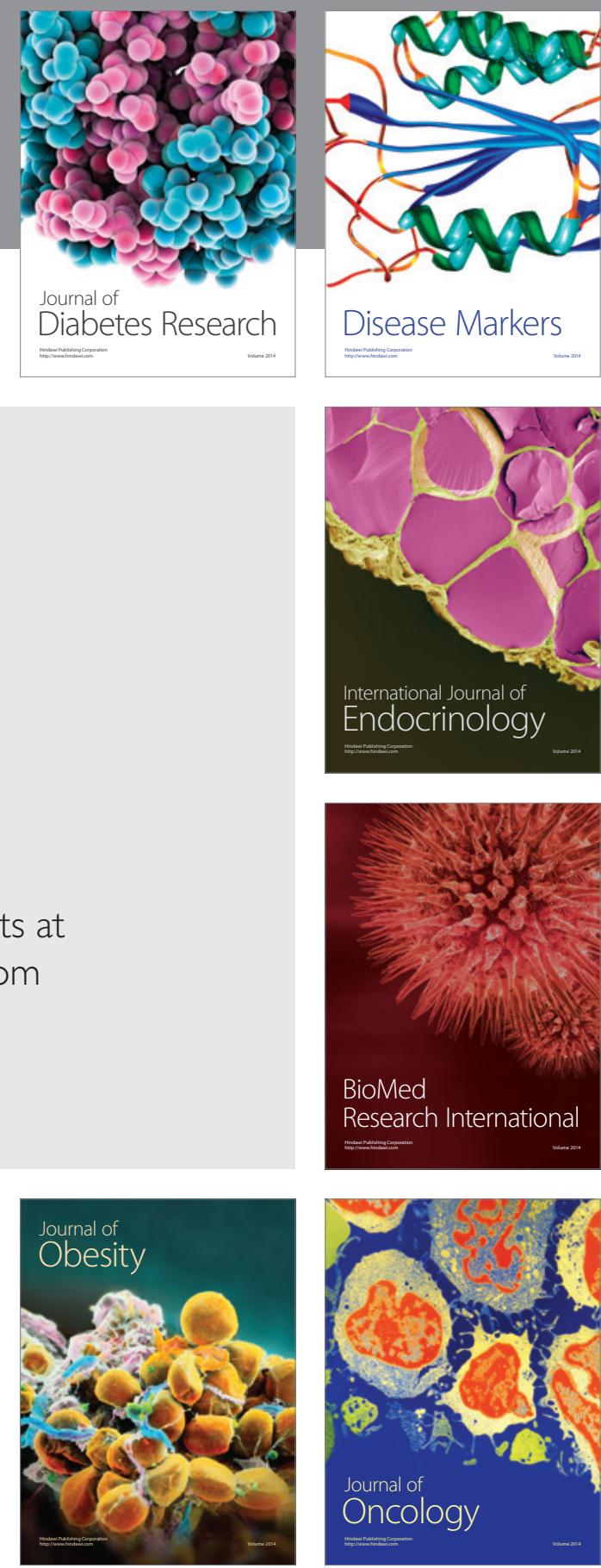

Disease Markers
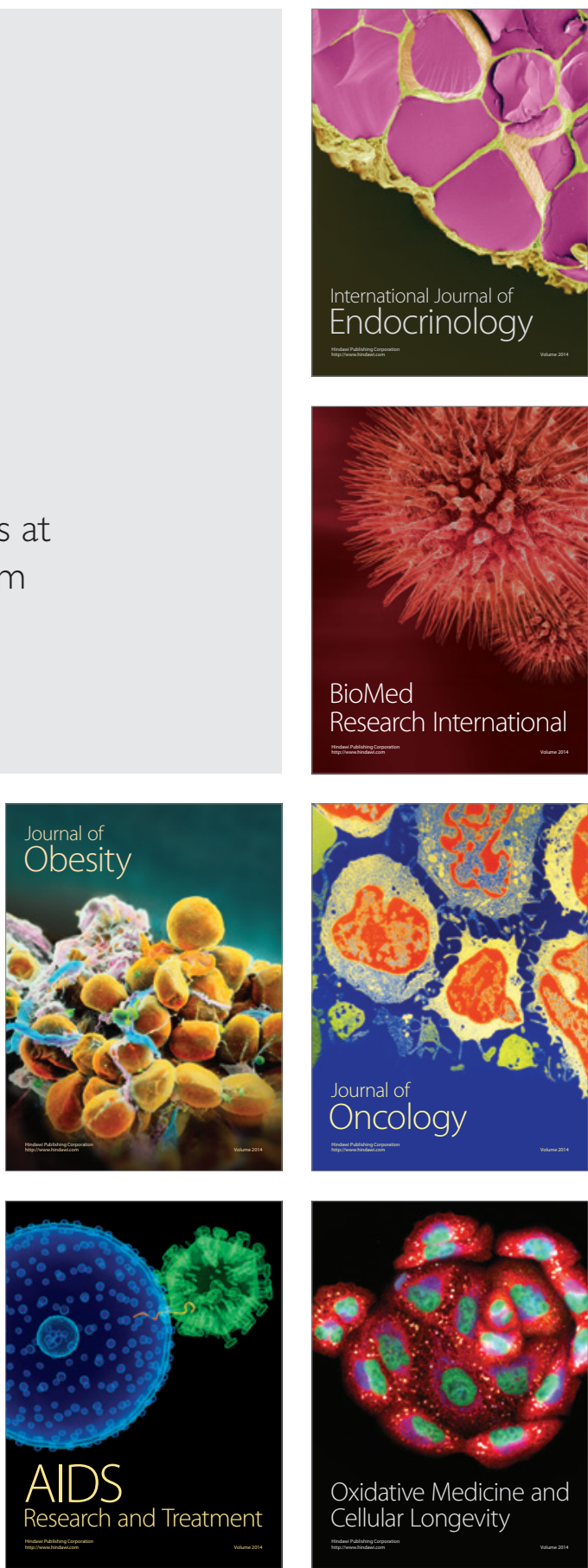